\title{
Drug delivery in overcoming the blood-brain barrier: role of nasal mucosal grafting
}

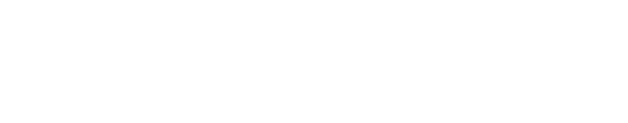

\author{
Carlotta Marianecci' \\ Federica Rinaldi ${ }^{2}$ \\ Patrizia Nadia Hanieh' \\ Luisa Di Marzio 3 \\ Donatella Paolino 4,5 \\ Maria Carafa' \\ 'Department of Drug Chemistry \\ and Technology, University of \\ Rome "Sapienza", Rome, Italy; \\ ${ }^{2}$ Center for Life Nano Science@ \\ Sapienza, Fondazione Istituto \\ Italiano di Tecnologia, Rome, Italy; \\ ${ }^{3}$ Department of Pharmacy, University \\ "G. d'Annunzio", Chieti, Italy; ${ }^{4}$ IRC \\ FSH-Interregional Research Center \\ for Food Safety \& Health, Campus \\ Universitario "S. Venuta", University of \\ Catanzaro "Magna Græcia”, Catanzaro, \\ Italy; ${ }^{5}$ Department of Health Sciences, \\ Campus Universitario "S. Venuta", \\ University of Catanzaro "Magna \\ Græcia", Catanzaro, Italy
}

\begin{abstract}
The blood-brain barrier (BBB) plays a fundamental role in protecting and maintaining the homeostasis of the brain. For this reason, drug delivery to the brain is much more difficult than that to other compartments of the body. In order to bypass or cross the $\mathrm{BBB}$, many strategies have been developed: invasive techniques, such as temporary disruption of the BBB or direct intraventricular and intracerebral administration of the drug, as well as noninvasive techniques. Preliminary results, reported in the large number of studies on the potential strategies for brain delivery, are encouraging, but it is far too early to draw any conclusion about the actual use of these therapeutic approaches. Among the most recent, but still pioneering, approaches related to the nasal mucosa properties, the permeabilization of the BBB via nasal mucosal engrafting can offer new potential opportunities. It should be emphasized that this surgical procedure is quite invasive, but the implication for patient outcome needs to be compared to the gold standard of direct intracranial injection, and evaluated whilst keeping in mind that central nervous system diseases and lysosomal storage diseases are chronic and severely debilitating and that up to now no therapy seems to be completely successful.
\end{abstract}

Keywords: CNS, BBB, nasal mucosa, grafting, lysosomal storage diseases, enzyme replacement therapies

\section{Introduction}

There are more than 1,000 agents which are active on central nervous system (CNS) pathologies that have shown promising perspectives in preclinical studies but failed to show good results in the later phases of the development process (at Phase III or even after registration). ${ }^{1-5}$

For this reason, disorders of the CNS represent one of the largest areas of unsatisfied medical needs, with about 2 billion people affected worldwide and many pharma companies moving away from such fields of innovative research because of the high risk of failure associated with CNS medicines, together with the ever increasing approval time. . $^{6}$

One of the major reasons for the poor translation of neuroscience research into medicines is the high degree of complexity of the human CNS, and effective noninvasive treatment of neurological diseases is often limited by the very limited access of therapeutic agents into the CNS owing to the presence of two anatomical and biochemical dynamic barriers: the blood-brain barrier (BBB) and the blood-cerebrospinal fluid barrier (BCSFB). ${ }^{8}$

It is already well known that BBB is formed by endothelial cells (ECs), which line up in capillaries of the brain and spinal cord by a variety of pericytes, vascular smooth muscle cells, astrocytes, and microglia. ${ }^{9}$ The BBB, composed of densely packed cells
Correspondence: Maria Carafa

Department of Drug Chemistry and

Technology, University of Rome

"Sapienza", Piazzale Aldo Moro 5,

00185 Rome, Italy

$\mathrm{Tel}+390649913603$

Fax +390649913133

Email maria.carafa@uniromal.it
Drug Design, Development and Therapy 2017:I I 325-335

325 
with copious intercellular tight junctions (TJs), active efflux pumps, and a trilamellar basement membrane, regulates the synaptic signaling function. Furthermore, BBB protects the CNS from neurotoxic substances, ensures brain nutrition, and prevents the entry of unwanted cells into the brain as well as the absorption of polar or high molecular weight (HMW) molecules larger than $500 \mathrm{Da}$; in other words, more than $98 \%$ of therapeutic drugs. ${ }^{10-12}$

The BBB also displays immunological and transportation features. The immune barrier is composed of microglia, perivascular mast cells, and macrophages. The transport barrier includes para- and transcellular routes. In addition, the brain is extremely rich in vascularity, with each neuron in contact with a capillary. ${ }^{13-15}$

The BCSFB is formed by the TJs of choroid plexus cells surrounding the microvascular endothelium with intracellular gap and fenestration. Owing to their structural difference, $\mathrm{BBB}$ and BCSFB have different main functions, but they both participate in controlling the transfer of molecules between the blood and the brain parenchyma or cerebrospinal fluid (CSF).

Under certain physiological conditions, circulating molecules can only gain access to the brain or CSF via a transcellular route through the capillary endothelial cells or choroid plexus cells, by either passive or active transport, or both.

Furthermore, if molecules overcome the BBB, they are not able to do so in high amounts, and they are exposed to degradation and/or rapid efflux from the CNS. ${ }^{16}$

Different transporters suitable for targeting molecules and delivering endogenous and exogenous compounds across the $\mathrm{BBB}$ are located on the BBB (Figure 1).
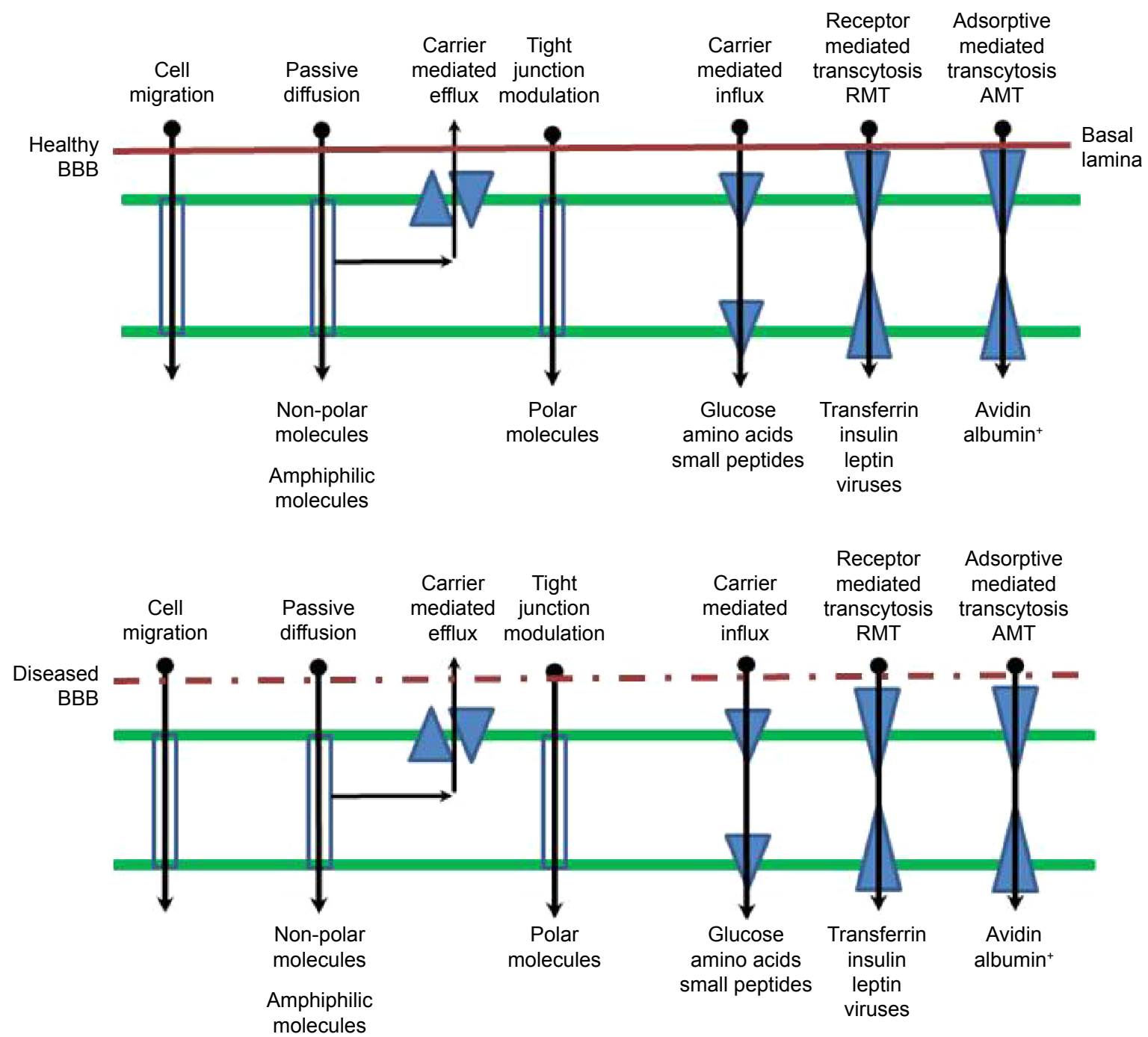

Figure I Transport routes across the blood-brain barrier.

Note: Green lines indicate tight junctions; blue boxes indicate no carrier or receptor mediated transport.

Abbreviations: BBB, blood-brain barrier; RMT, receptor-mediated transcytosis; AMT, adsorptive-mediated transcytosis. 
All these highly regulated and efficient features supply BBB with multiple functions as a physical barrier (TJ), a transport barrier (P-gp), a metabolic or enzymatic barrier, and an immunological barrier. ${ }^{17,18}$

Functions and organization of the BBB can be altered under pathological conditions, such as multiple sclerosis, epilepsy, acquired immune deficiency syndrome (AIDS), dementia, stroke, and brain cancer. ${ }^{19,20}$

It should also be pointed out that alterations in the barrier, as in the blood-brain tumor barrier (BBTB), often form an increased obstacle in CNS therapy by preventing the delivery of potentially effective therapeutic agents. ${ }^{21}$

Molecular and physiological mechanisms involved in the transport of compounds through the BBB can be used to design drug and drug carriers for brain delivery.

Ligands such as peptides, monoclonal antibodies (mAbs), modified proteins, peptidomimetic antibodies, and penetrating peptides can be conjugated to bioactive compounds or to colloidal supramolecular aggregates and potentially used to cross the BBB and accumulate inside the CNS. ${ }^{22}$

The paradox is that more than $99 \%$ of the global CNS drug development effort is devoted to CNS drug discovery, while less than $1 \%$ is devoted to CNS drug delivery. ${ }^{23}$

Fortunately, scientists realized how important is the pathway from the systemic circulation to the CNS, and a few years ago, the Stroke Therapy Academic Industry Roundtable Preclinical Recommendations group highly recommended that future neurological disease research should consider also the method of drug delivery when developing novel drugs. ${ }^{24}$

\section{Current methodologies for drug delivery across the $B B B$}

In order to bypass or cross the BBB, many strategies have been developed: invasive techniques, such as temporary disruption of the $\mathrm{BBB}$ or intraventricular and intracerebral direct administration of the drug, and noninvasive techniques. The invasive strategies, however, compromise the integrity and the functions of the BBB, allowing the potential accumulation of neurotoxic xenobiotics and exogenous agents; a state that is moderately to severely neurotoxic. ${ }^{25,26}$

Noninvasive chemical approaches involve the "manipulation" of active substances to be administered with the aim of increasing physiological stability and degree of penetration into the brain by passive diffusion or by active targeting. For this purpose, several methods have been proposed, such as the lipidization via addition of lipid-like molecules to the structure; the addition of molecules able to use specific endogenous transporters (eg, nutrients and other essential compounds), and the synthesis of prodrugs. Chemical modifications can be considered a safe, noninvasive approach, but they need chemical or enzymatic in vivo transformations that sometimes may lead to the loss of therapeutic activity or the activation of the physiological defense mechanisms of the membrane pumping out exogenous compounds.

Along with chemical modifications, there is an increase in multidisciplinary investigations, intended to facilitate the crossing of BBB, that combine biological, nanotechnological, and even biophysical expertise. In this sense, the most recent approaches concern TJ opening (eg, through the utilization of compounds like bradykinin via second messengers), receptor-mediated, adsorptivemediated transportation (some potentially useful technologies based on receptor-mediated transcytosis are currently under clinical evaluation for brain tumor therapy Clinicaltrials.gov: NCT01967810, NCT02048059, NCT01480583, NCT01386580, NCT01818713), and efflux pump inhibition by specific inhibitors that appears to be a strategy capable of delivering the drug to the brain without affecting the integrity of the endothelial layer and $\mathrm{TJ}$ that might cause toxicities. Also, energy-based physical methods, such as ultrasound, microwave, or electromagnetic fields, are under evaluation.

Another approach for the improvement of brain targeting is represented by the combination of the drug with the living cells, acting as Trojan horses that can cross the BBB..$^{27-29}$

In recent times, much attention has been given to nanotechnology in many areas because of its significant potential for the successful treatment of severe diseases such as cancer and neurological diseases.

A promising noninvasive approach to brain delivery involves nanomedicine, which takes advantage of the possible assembly of several biomaterials that can provide a delivery platform, at nanoscale size, capable of raising brain levels of drug substances otherwise unable to cross the BBB. ${ }^{23,30}$

The success of a therapeutic strategy by means of nanocarriers depends on their ability to entrap drugs, to penetrate through anatomical barriers, and to release the incorporated drugs, accompanied by a good stability in the nanometric size range. ${ }^{26}$

One possible strategy is the drug encapsulation into brain-targeted nanocarriers such as polymeric nanoparticles, dendrimers, vesicular carriers, metal, and silicon. These nanocarriers can have an organic or inorganic core and a surface coating with organic moieties able to interact 
with the biological system at a molecular level for crossing the BBB. ${ }^{31}$

Modification of nanocarrier surface by adsorption or covalent linking of hydrophilic polymers, such as PEG, polysorbate 80 , or polysaccharides, leads to an increase in nanocarrier circulation in the blood, whereas the surface derivatization with molecules that recognize cellular receptors facilitates the penetration of nanoparticles through the BBB. ${ }^{32}$

The strategy often termed active targeting involves two types of transports: adsorptive-mediated transcytosis (AMT) and receptor-mediated transcytosis (RMT). The AMT has gained considerable attention because several studies assess that this strategy has the possibility to enhance the transport of nanocarriers across the $\mathrm{BBB}$, using cationic proteins or cell-penetrating peptides. ${ }^{33}$ However, as AMT is a nonspecific process, conjugation of such cationic proteins will also increase the adsorptive uptake process of nanocarriers in other parts of the body, which may possibly create toxic and immunogenic concerns. Transport of nanocarriers to the brain using the RMT process is more specific than AMT. The RMT involves addition of endogenous molecules on the nanocarrier surface, which are substrates for specific receptors expressed on the BBB. Addition of proteins (eg, transferrin, lactoferrin, Apolipoprotein E [ApoE]); peptides (eg, glutathione); or antitransferrin receptor antibody OX26 on the surface of liposomes, polymeric nanoparticles, and lipidic nanoparticles significantly increased the BBB penetrations of such nanocarriers. ${ }^{34}$

Size of the nanocarrier along with surface charge, surface hydration, and targeting strategy are important characteristics for development of a successful brain-targeted nanocolloid drug delivery system for glioblastoma multiforme (GBM) treatment. Among various possible nanocarriers, liposomes, polymeric nanoparticles, and lipid nanocarriers are the most widely studied, and will be discussed in detail in the following sections.

Preliminary results reported in the large number of studies on the potential strategies to overcome and/or cross the BBB are encouraging, but it is far too early to draw any conclusion about the actual practical applications of these therapeutic approaches.

\section{Challenge in nasal delivery to CNS}

Nasal delivery has conventionally been restricted to topically/ locally acting therapeutic agents for the treatment of nasal problems (eg, cold and nasal hypersensitivity). Recently, nasal route received increased attention as a substitute for oral and parenteral routes for several systemic therapeutic agents. The highly vascularized and immunogenic nasal mucosa allows fast onset of action, enhanced bioavailability and patient compliance. ${ }^{35-37}$

The pioneering method of intranasal delivery for drug delivery into the CNS was first described in 1991 by Dr William H Frey, and nowadays, it has proven to be a safe and efficient way to deliver exogenous molecules to the CNS. ${ }^{38-42}$ After nasal administration for CNS delivery, four physiological steps are possible, and they include the olfactory nerve pathway, trigeminal nerve pathway, vascular pathway, and the lymphatic pathway. ${ }^{43}$

During the last two decades, four areas, including intranasal delivery validation, pathway elucidation, intranasal delivery of various therapeutics for the treatment of neurological diseases, and enhancement of the efficiency of intranasal delivery, have been explored. ${ }^{44,45}$

Nasal cavity delivery and nasal absorption are promising approaches but are still uncertain. Direct intranasal transport is not always well established, and controversial results were obtained when similar substances were administered, and sometimes, even opposite results have been reported after nasal delivery to the CSF. ${ }^{46,47}$

A few years ago, Merkus and van den Berg, ${ }^{48}$ reviewing more than 100 papers, reported that no significant pharmacokinetic evidence was yet capable of establishing that, in humans, the intranasal route of administration actually provides enhanced targeting to the CNS, compared to the systemic route.

The real potential of the nasal route for drug delivery purposes clearly needs to be explored, developing new approaches and amending theories. ${ }^{37}$

Nasal mucosal properties (ie, permeability to very large and polar molecules), are used in recent but still pioneering approaches, and among the various techniques, the permeabilization of the BBB via nasal mucosal engrafting could offer new important opportunities.

\section{Nasal mucosal grafting: potential for drug delivery and implications for enzyme replacement therapies Nasal mucosal grafting}

Over the last few decades, the management of anterior skull base defects, CSF leaks, and encephaloceles by endoscopic, minimally invasive approaches is improving. Whether the etiology of these defects is spontaneous, traumatic, or surgical (eg, removal of brain tumors through the nose without facial incisions), the large majority of such defects can be repaired by means of free mucosal grafts that can be applied as a single layer reconstruction for small leaks or as a multilayer reconstruction for larger defects. ${ }^{49}$ 
Middle turbinate, inferior turbinate, and nasal septum can be used as donor sites for free mucosal grafts for endoscopic endonasal reconstruction of the skull base. In this sense, the harvest of a nasal floor free mucosal graft was recently proposed as a rapid, potentially less morbid method. ${ }^{50}$ Accessibility, ease of placement, and high take rate make free mucosal grafts suitable candidates for reconstruction of many skull base defects. ${ }^{51}$

The repairs by mucosal grafts are permanent, watertight, and immunocompetent, and they can also be used to replace large regions of the BCSFB within the arachnoid with relatively permeable mucosa. ${ }^{52,53}$ In principle, the engrafted mucosa can be dosed with therapeutic agents applied topically. Given the lack of underlying arachnoid membrane, these mucosal grafts, using purely autologous tissues, could be proposed to solve problems related to the $\mathrm{BBB}$ crossing by HMW or polar agents and a direct delivery to the brain and to the subarachnoid space (Figure 2).

This approach was tested for the first time by Bleier et $\mathrm{al}^{54}$ in a proof-of-concept extracranial graft model in mice. The applied mucosal grafting method was adapted by a surgical technique that is currently used in the field of endoscopic skull base surgery defects. ${ }^{55}$ The ability of septal mucosal grafts to allow diffusion of HMW markers into the CNS was investigated. A murine model, mimicking the human skull base, was developed and validated. A polypropylene reservoir, allowing topical dosing of the graft with different fluorescent markers $(20-500 \mathrm{kDa})$, was surgically implanted over the mucosal graft. The whole mucosal implant was well tolerated, and no evidence of subcutaneous infection or distress related to the surgical site was observed. Furthermore, the delivery via the mucosal graft was compared to the intranasal delivery, and a minimal delivery, similar to that of the negative control (dura membrane kept intact), was obtained. The direct exposure of the brain to the marker solution was used as positive control (no intervening dura or nasal mucosa). This study showed that the mucosal grafts allowed good permeability to HMW molecules and that the transport rate seemed to be molecular weight dependent. By extending the exposure duration, this effect was partially overcome. By developing different drug eluting polymers, the release rate can be modulated over time, and this could be a great challenge in combining innovative surgical and technological approaches. ${ }^{54}$

The interesting findings of this study are limited by the possibility of comparing an animal model to a clinical setting owing to regional differences in convection throughout the murine and human brain. First of all, in humans, the ratio between mucosal graft area and brain volume ratio is much higher. In addition, in the clinical application, diffusion to more distal regions of the brain can be possible via CSF circulation, while it should be quite impossible in the murine model because of the occlusion of the smaller subarachnoid space after craniotomy.

Despite the aforementioned limitations of nasal mucosal grafting, the delivery of glial-derived neurotrophic factor (GDNF) was investigated in a mouse model for the treatment of Parkinson disease (PD). ${ }^{56}$ In this study, a murine 6-hydroxydopamine PD model was applied. A parietal craniotomy and arachnoid defect was repaired with a heterotopic donor mucosal graft. Given the permeability of these mucosal grafts to HMW molecules, as previously reported, the possibility, by means of this approach, to

\section{Normal structure}

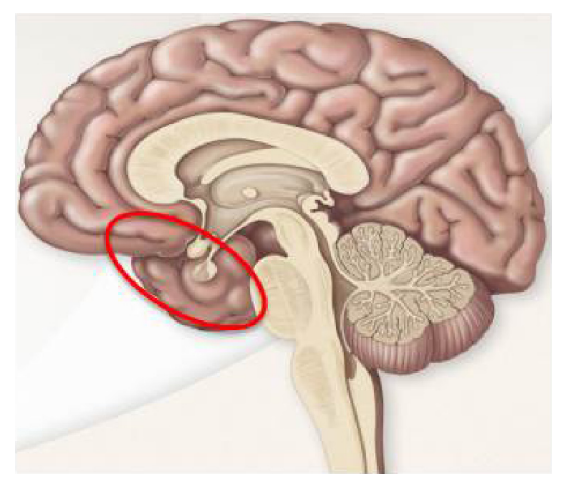

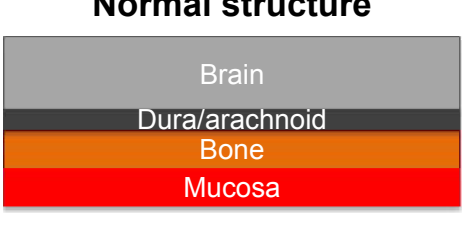

After mucosal grafting

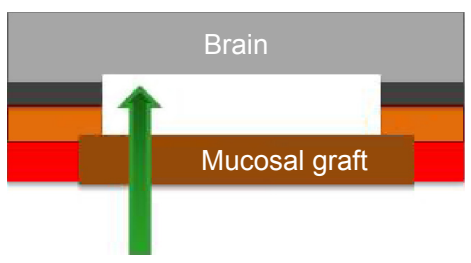

Figure 2 Graphic description of nasal mucosa graft.

Notes: The area circled in red indicates the nasal mucosal grafting area; the green arrow indicates absorption direction; and the white boxed area indicates removed bone and dura/arachnoid. 
bypass the BBB and to deliver HMW neuroactive substances, engrafted over an arachnoid defect, directly to the CNS was evaluated. ${ }^{54}$

GDNF was released by a polypropylene reservoir placed over the mucosal graft. Cyanoacrylate adhesive was used to attach the reservoir to the skull, and dental cement was applied to the skull. To maintain structural integrity, two bone screws were implanted into the skull before cement application.

GDNF was delivered through the mucosal graft, and the therapeutic efficacy was evaluated and compared with direct intrastriatal GDNF injection using behavioral assays (rotarod and apomorphine rotation). To compare the preservation of substantia nigra cell bodies, an immunohistological analysis was also used. Both behavioral and histological results demonstrated the therapeutic efficacy of transmucosal GDNF delivery. No significant difference was reported in neuronal survival between the transmucosal and injected GDNF mice, suggesting that the transmucosal pathway could be as effective as direct intraparenchymal injection in GDNF delivering to the end-target structures deep within the brain. Furthermore, the proposed mucosal grafting method could show advantages over the simple intranasal drug delivery while avoiding the concerns over the limitations of olfactory uptake in humans. In principle, the graft may be placed adjacent to the sphenoid sinus, which creates an intrinsic reservoir capable of retaining a greater volume of any intranasally applied solution. This reservoir can also help to enhance mucosal residence time by limiting the immediate clearance of the drug from the nose because of the mucociliary clearance. ${ }^{57}$

This chirurgical procedure can be an efficient method to assure drug localization in the CNS, especially in the case of HMW drugs, such as enzymes that are widely used in the treatment of lysosomal storage diseases.

The local effect of this surgical procedure is the replacement of $\mathrm{BBB}$ and $\mathrm{BCSFB}$, while the effect on the remaining part of $\mathrm{BBB}$ and $\mathrm{BCSFB}$ has not been evaluated and is not easily predictable by the results reported in the three cited research papers. Furthermore, the long-term effects of grafting nasal mucosa have not yet been evaluated because this technique has only been reported in a few very recent experimental studies on mice. ${ }^{54-56}$

\section{Lysosomal storage diseases}

Lysosomal storage diseases (LSDs) include more than 50 inherited metabolic disorders characterized by the intralysosomal accumulation of undegraded substrates. Chemical properties of the accumulated substrate allow classification of the different types of LSDs. Individually, these disorders are rare, but their cumulative prevalence is relatively high when compared with other groups of rare diseases, and they reach an incidence of one over 8,000 newborns. ${ }^{58}$

LSDs are responsible for various clinical consequences on multiple organs and systems with visceral, ocular, hematological, skeletal, and neurological manifestations, and there is partial phenotypic overlap among different disorders. Symptoms may emerge in utero, during the newborn period, or in late adulthood. About $75 \%$ of LSD patients present a neurological impairment.

LSDs are often responsible for physical and neurological disabilities, and they can interfere with patients' health and life expectancy. Therefore, the LSD therapy requires a multidisciplinary collaboration.

LSDs are caused by mutations in genes encoding soluble acidic hydrolases, integral membrane proteins, activator proteins, transporter proteins, or nonlysosomal proteins that are necessary for the lysosomal functions. These deficiencies are responsible for intralysosomal accumulation of undegraded substrates in multiple tissues and organs.

Perturbation of lysosomal function may also lead to less obvious consequences, such as $\mathrm{PD}$, which is the prevalent neurodegenerative disorder. Histopathologically, PD is characterized by the accumulation of insoluble aggregates of the presynaptic protein $\alpha$-synuclein in typical intraneuronal inclusions (Lewy bodies), by the selective loss of dopaminergic neurons in the substantia nigra, and clinically by movement and postural defects. Although the mechanisms underlying this connection have not been fully elucidated, dysfunctions in several lysosomal proteins (and lysosomal gene mutations) have been involved in the pathogenesis of PD.

Mutations in gene encodings, essential components of the endolysosomal-autophagic pathway, have also been described in other neurodegenerative diseases, including Alzheimer disease, Huntington disease, frontotemporal dementia, and Charcot-Marie-Tooth type 2B.

In the past 25 years, much effort has been directed at developing specific therapies to correct the metabolic defects of these disorders by means of different strategies that were intended to target a specific event in the pathogenetic cascade, increasing the activity of the defective enzyme or protein by different ways.

The normal enzyme, obtained by recombinant technology or its precursor secreted in the circulation by engineered cells, may be administered through the endocytic pathway, by 
intravenous administration. It is also possible to correct the gene mutation by delivering a wild-type copy of the mutated gene or to protect from degradation the mutant enzyme by increasing its residual activity.

Other strategies, aimed at restoring the equilibrium between the synthesis of substrates and their degradation by lysosomal enzymes, have been studied. They include reduction of substrate synthesis, enhancement of the clearance of substrates from cells and tissues, and manipulation of specific cellular pathways, involved in vesicle trafficking (Figure 3). ${ }^{59}$

Moreover, alterations in the BBB are likely involved in LSDs as well as in all neurodegenerative diseases. ${ }^{60}$ Actually, neuroinflammatory changes present in neurodegeneration commonly affect the BBB or its function by altering transport systems, affecting the integrity of TJ, enhancing immune cell entry, or influencing the BBB's role as a signaling interface. ${ }^{61}$ In addition, ion balance, disruption of transport systems, and altered function of BBB constitutive enzymes $^{61,62}$ are also induced by BBB dysfunction. The BBB impairment influences drug therapy, in particular the process regulating the entry of drugs normally excluded from the brain. ${ }^{61,63}$

\section{Enzyme replacement therapy}

Enzyme replacement therapy (ERT) is one of the approaches for several LSD treatments. In the early 1990s the efficacy of ERT in Gaucher disease was demonstrated, and this approach has been used to treat other LSDs, including Fabry disease, Pompe disease, and Mucopolysaccharidosis I, II, and VI. ${ }^{64-71}$

After the conclusion of the first phase of ERT development, advantages and limitations of this approach have emerged. Important limitations include the reduced bioavailability of intravenously injected recombinant enzymes, and, therefore, strategies to improve ERT efficacy need to be identified. Recombinant enzymes are HMW molecules that are unlikely diffuse across membranes and are unable to reach therapeutic concentrations in some target tissues, particularly the brain. An additional major goal in future years should be the discovery of a BBB crossing enzyme because two-thirds of LSDs are involved in neurological symptoms and progressive neurodegeneration.

Strategies to improve the delivery of enzymes to the CNS are currently undergoing preclinical and clinical evaluation. For example, $\beta$-glucuronidase, which is deficient in Mucopolysaccharidosis VII, has been chemically modified to

\section{LSDs management}

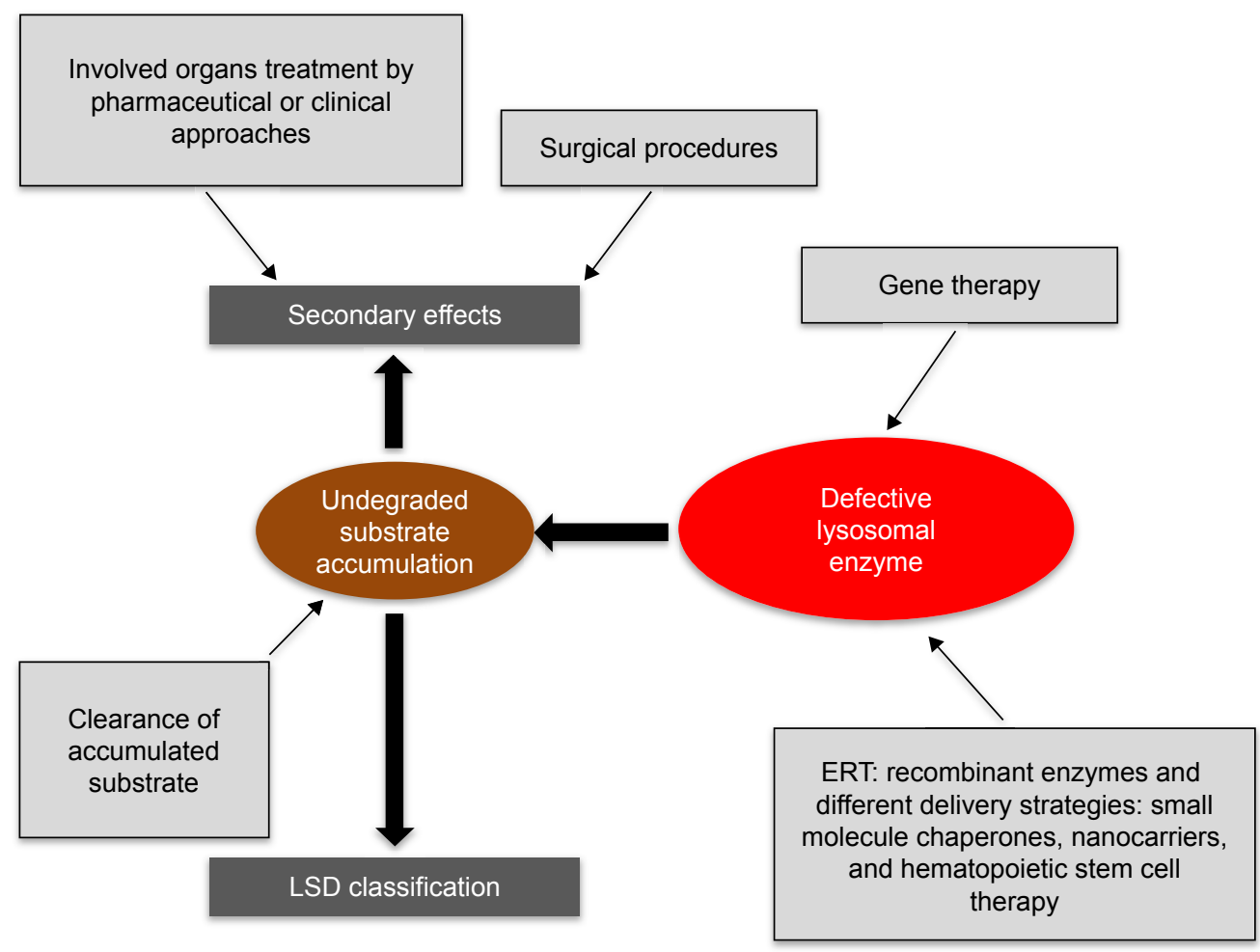

Figure 3 Schematic representation of therapeutic approaches to LSDs. Abbreviations: LSDs, lysosomal storage diseases; ERT, enzyme replacement therapy. 
increase its plasma half-life and facilitate its traffic through the BBB. ${ }^{72,73}$ Other approaches consider the use of so-called Trojan horses, made of chimeric enzymes conjugated with peptides that can allow penetration through the BBB leading to brain delivery by specific pathways such as the apolipoprotein and receptor pathways. These approaches have been evaluated in preclinical studies for $\alpha$-iduronidase, iduronate2-sulfatase, arylsulfatase A, and tripeptidyl peptidase I. ${ }^{74-78}$

In addition to these approaches, preclinical studies are evaluating the use of invasive procedures to deliver recombinant enzymes directly into the CSF for the treatment of several lysosomal disorders. Intrathecal and lumbar or cisterna magna punctures have been studied for the administration of various types of mucopolysaccharidoses in animal models. ${ }^{79-82}$ Intrathecal ERT has been translated into human therapy for mucopolysaccharidoses types I and VI. Devices for continuous intrathecal infusion have been developed and tested in preclinical studies. Some clinical trials of intrathecal administration of ERT are ongoing, and others have been completed. Invasive techniques for BBB crossing were based on neurosurgery or on a temporary chemical/physical disruption of the barrier, produced by biochemical and immunological changes or by an osmotic shift. ${ }^{83-85}$ However, both these approaches entail several drawbacks such as the invasiveness and the high costs of neurosurgery, the physiological stress, or the transient increase in the intracranial pressure, along with high risk of infections and damages from toxins, due to the BBB temporary opening. Therefore, to improve drug delivery to the brain, noninvasive techniques have been explored, and among them the nanotechnology-based approach surely represents one of the most encouraging procedures.

To obtain a selective targeting to receptors highly expressed on the BBB, several possible ligands have been identified including transferrin, lactoferrin, insulin and leptin, and the LDL-receptor related protein LRP1. ${ }^{11}$ The proposed nanocarriers include a wide variety of drug-delivery vehicles, including dendrimers, micelles, liposomes, nanoscale ceramics, and polymer nanoparticles. ${ }^{86-88}$ Different carriers offer different approaches to enzyme delivery. Liposomes are the first generation of nanoparticulate drug-delivery systems that reached the market, and they consist of one or more phospholipid bilayers delimiting an internal aqueous compartment. They are able to deliver hydrophobic, hydrophilic, and amphiphilic molecules. It has been reported that $\beta$-galactosidase loaded liposomes injected by rat tail vein can penetrate the $\mathrm{BBB}$ and reach the lysosomes in the CNS more efficiently than the free enzyme. ${ }^{89}$ Similar results have been obtained by ex vivo and in vivo studies on saposin C-loaded liposomes that improve the neurological condition. ${ }^{90}$ Liposomes modified with lysosomotropic octadecyl-rhodamine B (Rh) and loaded with therapeutic glucocerebroside velaglucerase $\alpha$ (VPRIVTM) increase lysosomal delivery of the enzyme into Gaucher cells. ${ }^{91}$ Biodegradable and biocompatible nanoparticles, composed of poly (D,L-lactide-co-glycolide) (PLGA), have been investigated for recombinant proteins, plasmid DNA, and low molecular weight compounds brain delivery. ${ }^{92}$ Recently, in vitro and in vivo animal studies demonstrated the ability of PLGA nanoparticles, modified with glycopeptides (g7-NPs), to efficiently cross the BBB and to be transported intra- and intercellularly within vesicles for a more effective treatment of neurological disorders. ${ }^{93-95}$ Pegylated immunoliposomes, derivatized with the 8D3 antitransferrin receptor antibody, have been used to target the luciferase and $\alpha$-galactosidase genes into the rat brain and to assure their activity after intracellular delivery.

Systemic gene therapy, together with several recombinant vector systems, is under evaluation and appears to be promising. ${ }^{96,97}$ Finally, it was shown that hemopoietic stem cells, derived from the monocyte/macrophage lineage and genetically modified to produce replacement enzymes, are capable of crossing the BBB in rodent models and entering into the CNS. ${ }^{98,99}$ However, so far neither nanocarriers nor adeno-associated virus vectors nor cell-based therapies capable of crossing the $\mathrm{BBB}$ have reached clinical trials in LSD, for both technological and toxicological problems.

\section{Conclusion}

The BBB plays a fundamental role in protecting and maintaining the homeostasis of the brain. For this reason, drug delivery to the brain is much more difficult than to other compartments of the body. The brain capillary endothelium excludes from the brain around 100\% of large-molecule and more than $98 \%$ of small-molecule drugs.

Consequently, possible therapies with charged or macromolecular drugs, which could be capable of preventing or even reversing certain neurologic diseases, are actually clinically ineffective owing to their inability to cross the BBB. This is the central and crucial problem for an effective treatment of both LSDs and the associated neuropathology.

The fact that therapeutic approaches capable of modifying the natural evolution of many LSDs in the peripheral organs have been published and/or are under development (eg, enzyme replacement therapy, nanotechnology, gene therapy, and the use of stem cells) makes LSDs the ideal 
environment in which to further investigate the relationship between lysosomal dysfunction-related neurodegeneration and the development of new strategies capable of crossing the BBB and reaching the brain compartment.

A number of studies showed that, in neurodegenerative diseases and LSDs, the function and integrity of the BBB and, in particular, of TJ, might be compromised. In these conditions, an increased passive extravasation of solutes, including proteins up to at least a molecular weight of $150 \mathrm{kDa}$, into the brain can be demonstrated. In general, damage to the BBB can induce even worse CNS damages. Furthermore, the neuropathology, related to the accumulation of storage products, and the correlation between the two processes, needs to be better understood.

It must be clarified that the ERT treatment or other approaches must bypass the BBB barrier function. At present, the best way to reach the $\mathrm{CNS}$ crossing the $\mathrm{BBB}$, whether by a clinical approach or a technological one using innovative drug delivery systems, is not clearly defined. Valid results obtained on a large-animal are still not available and the nasal mucosal grafting approach appears not yet feasible for clinical translation.

Mucosal grafting requires an endoscopic procedure, and this can seriously limit patient compliance. However, because the grafting technique is adapted from an existing endoscopic surgical procedure, its safety profile has been well established over several decades of clinical use and is associated with a lower morbidity rate than other currently accepted methods of BBB invasive penetration. It should be emphasized that this surgical procedure is quite invasive, but at present, patients are subjected to direct intracranial injection that is not always tolerated, because neurodegenerative diseases and LSDs are chronic and severely debilitating diseases, and to this day no therapy seems to be completely successful. ${ }^{100-102}$

\section{Disclosure}

The authors report no conflicts of interest in this work.

\section{References}

1. Pardridge WM. Why is the global CNS pharmaceutical market so underpenetrated? Drug Discov Today. 2002;7(1):5-7.

2. Nutt D, Goodwin G. ECNP summit on the future of CNS drug research in Europe 2011: report prepared for ECNP. Eur Neuropsychopharmacol. 2011;21(7):495-499.

3. Turner RC, Dodson SC, Rosen CL, Huber JD. The science of cerebral ischemia and the quest for neuroprotection: navigating past failure to future success. J Neurosurg. 2013;118(5):1072-1085.

4. Garg T, Bhandari S, Rath G, Goyal AK. Current strategies for targeted delivery of bio-active drug molecules in the treatment of brain tumor. J Drug Target. 2015;23(10):865-887.
5. Iqbal K, Liu F, Gongm C. Tau and neurodegenerative disease: the story so far. Nat Rev Neurol. 2016;12(1):15-27.

6. Palmer AM, Alavijeh MS. Translational CNS medicines research. Drug Discov Today. 2012;17(19-20):1068-1078.

7. Pangalos MN, Schechter LE, Hurko O. Drug development for CNS disorders: strategies for balancing risk and reducing attrition. Nat Rev Drug Discov. 2007;6(7):521-532.

8. Gaudin A, Andrieux K, Couvreur P. Nanomedicines and stroke: toward translational research. J Drug Deliv Sci Tec. 2015;30(B):278-299.

9. Bernacki J, Dobrowolska A, Nierwinska K, Malecki A. Physiology and pharmacological role of the blood-brain barrier. Pharmacol Rep. 2008;60(5):600-622.

10. Cardoso FL, Brites D, Brito MA. Looking at the blood-brain barrier: molecular anatomy and possible investigation approaches. Brain Res Rev. 2010;64(2):328-363.

11. Abbott NJ, Patabendige AA, Dolman DE, Yusof SR, Begley DJ. Structure and function of the blood-brain barrier. Neurobiol Dis. 2010; 37(1):13-25.

12. Pardridge WM. Drug transport across the blood-brain barrier. J Cereb Blood Flow Metab. 2012;32(11):1959-1972.

13. Aguzzi A, Barres B, Bennett ML. Microglia: scapegoat, saboteur, or something else? Science. 2013;339(6116):156-161.

14. Daneman R, Rescigno M. The gut immune barrier and the blood-brain barrier: are they so different? Immunity. 2009;31(5):722-735.

15. Banks WA. Blood-brain barrier as a regulatory interface. Forum Nutr. 2010;63:102-110.

16. Chen Y, Dalwadi G, Benson HA. Drug delivery across the blood-brain barrier. Curr Drug Deliv. 2004;1(4):361-376.

17. Pardridge WM. Molecular biology of the blood-brain barrier. Mol Biotechnol. 2005;30(1):57-70.

18. Abbott NJ, Ronnback L, Hansson E. Astrocyte-endothelial interactions at the blood-brain barrier. Nat Rev Neurosci. 2006;7(1):41-53.

19. de Vries HE, Kooij G, Frenkel D, Georgopoulos S, Monsonego A, Janigro D. Inflammatory events at blood-brain barrier in neuroinflammatory and neurodegenerative disorders: implications for clinical disease. Epilepsia. 2012;53(6):45-52.

20. Obermeier B, Daneman R, Ransohoff RM. Development, maintenance, and disruption of the blood-brain barrier. Nat Med. 2013;19(12): 1584-1596.

21. van Tellingend $\mathrm{O}$, Yetkin-Arikc B, de Gooijerd MC, Wesselinge P, Wurdinger T, de Vries HE. Overcoming the blood-brain tumor barrier for effective glioblastoma treatment. Drug Resist Updat. 2015;19:1-12.

22. Vlieghe P, Khrestchatisky M. Medicinal chemistry based approaches and nanotechnology-based systems to improve CNS drug targeting and delivery. Med Res Rev. 2013;33(3):457-516.

23. Aparicio-Blanco J, Martín-Sabroso C, Torres-Suarez AI. In vitro screening of nanomedicines through the blood brain barrier: a critical review. Biomaterials. 2016;103:229-255.

24. Fisher M, Feuerstein G, Howells DW, et al. Update of the stroke therapy academic industry roundtable preclinical recommendations. Stroke. 2009;40(6):2244-2250.

25. Calias P, Banks WA, Begley D, Scarpa M, Dickson P. Intrathecal delivery of protein therapeutics to the brain: a critical reassessment. Pharmacol Ther. 2014;144(2):114-122.

26. Celia C, Cosco D, Paolino D, Fresta M. Nanoparticulate devices for brain drug delivery. Med Res Rev. 2011;31(5):716-756.

27. Stojanov K, Zuhorn IS, Dierckx RA, de Vries EF. Imaging of cells and nanoparticles: implications for drug delivery to the brain. Pharm Res. 2012;29(12):32313-32340.

28. Chen Y, Liu L. Modern methods for delivery of drugs across the blood-brain barrier. Adv Drug Deliv Rev. 2012;64(7):640-665.

29. Hersh DS, Wadajkar AS, Roberts NB, et al. Evolving drug delivery strategies to overcome the blood brain barrier. Curr Pharm Des. 2016; 22(9):1177-1193.

30. Banks WA. From blood-brain barrier to blood-brain interface: new opportunities for CNS drug delivery. Nat Rev Drug Discov. 2016; 15(4):275-292. 
31. Singh D, Kapahi H, Rashid M, Prakash A, Majeed AB, Mishra N. Recent prospective of surface engineered nanoparticles in the management of neurodegenerative disorders. Artif Cells Nanomed Biotechnol. 2016;44(3):780-791.

32. Ingallina $\mathrm{C}$, Rinaldi $\mathrm{F}$, Bogni $\mathrm{A}$, et al. Niosomal approach to brain delivery: development, characterization and in vitro toxicological studies. Int J Pharm. 2016;511(2):969-982.

33. Herve F, Ghinea N, Scherrmann JM. CNS delivery via adsorptive transcytosis. AAPS J. 2008;10(3):455-472.

34. Bhaskar S, Tian F, Stoeger T, et al. Multifunctional Nanocarriers for diagnostics, drug delivery and targeted treatment across blood-brain barrier: perspectives on tracking and neuroimaging. Part Fibre Toxicol. 2010;7:3.

35. Illum L. Nasal drug delivery - recent developments and future prospects. J Control Release. 2012;161(2):254-263.

36. Hansen K, Kim G, Desai KG, et al. Feasibility investigation of cellulose polymers for mucoadhesive nasal drug delivery applications. Mol Pharm. 2015;12(8):2732-2741.

37. Kumar A, Pandey AN, Jain SK. Nasal nanotechnology: revolution for efficient therapeutics delivery. Drug Deliv. 2016;23(3):671-683.

38. Frey WH. Neurologic Agents for Nasal Administration to the Brain. Emeryville, CA: Chiron Corporation; 1991.

39. Zhu J, Jiang Y, Xu G, Liu X. Intranasal administration: a potential solution for cross-BBB delivering neurotrophic factors. Histol Histopathol. 2012;27:537-548.

40. Jiang Y, Zhu J, Xu G, Liu X. Intranasal delivery of stem cells to the brain. Expert Opin Drug Deliv. 2011;8(5):623-632.

41. Lochhead JJ, Thorne RG. Intranasal delivery of biologics to the central nervous system. Adv Drug Deliv Rev. 2012;64(7):614-628.

42. Marianecci C, Rinaldi F, Hanieh PN, Paolino D, Di Marzio L, Carafa M. Nose to brain delivery: new trends in amphiphile-based "soft" nanocarriers. Curr Pharm Des. 2015;21(36):5225-5232.

43. Chen M, Wu B, Ye X, et al. Association between plasma homocysteine levels and obstructive sleep apnoea in patients with ischaemic stroke. J Clin Neurosci. 2011;18(11):1454-1457.

44. Pires A, Fortuna A, Alves G, Falcão A. Intranasal drug delivery: how, why and what for? J Pharm Pharm Sci. 2009;12(3):288-311.

45. Jiang Y, Li Y, Liu X. Intranasal delivery: circumventing the iron curtain to treat neurological disorders. Expert Opin Drug Deliv. 2015; 12(11):1717-1725.

46. Born J, Lange T, Kern W, McGregor GP, Bickel U, Fehm HL. Sniffing neuropeptides: a transnasal approach to the human brain. Nat Neurosci. 2002;5(6):514-516.

47. Merkus P, Guchelaar HJ, Bosch DA, Merkus FW. Direct access of drugs to the human brain after intranasal drug administration? Neurology. 2003;60(10):1669-1671.

48. Merkus FW, van den Berg MP. Can nasal drug delivery bypass the blood-brain barrier?: questioning the direct transport theory. Drugs R D. 2007;8(3):133-144.

49. Bleier BS, Wang EW, Vandergrift WA, Schlosser RJ. Mucocele rate following endoscopic skull base reconstruction using vascularized pedicled flaps. Am J Rhinol Allergy. 2011;25(3):186-187.

50. Suh JD, Ramakrishnan VR, DeConde AS. Nasal floor free mucosal graft for skull base reconstruction and cerebrospinal fluid leak repair. Ann Otol Rhinol Laryngol. 2012;121(2):91-95.

51. Ting JO, Metson R. Free graft techniques in skull base reconstruction. Adv Otorhinolaryngol. 2013;74(1):33-41.

52. Bernal-Sprekelsen M, Alobid I, Mullol J, Trobat F, Toma's-Barbera'n M. Closure of cerebrospinal fluid leaks prevents ascending bacterial meningitis. Rhinology. 2005;43(4):277-281.

53. Miyake MM, Bleier BS. The blood-brain barrier and nasal drug delivery to the central nervous system. Am J Rhin Allergy. 2015;29(2): 124-127.

54. Bleier BS, Kohman RE, Feldman RE, Ramanlal S, Han X. Permeabilization of the blood-brain barrier via mucosal engrafting: implications for drug delivery to the brain. PLoS One. 2013;8(4):e61694. 1-7.
55. Bleier BS, Palmer JN, Sparano AM, Cohen NA. Laser-assisted cerebrospinal fluid leak repair: an animal model to test feasibility. Otolaryngol Head Neck Surg. 2007;137:810-814.

56. Bleier BS, Kohman RE, Guerra K, et al. Heterotopic mucosal grafting enables the delivery of therapeutic neuropeptides across the blood brain barrier. Neurosurgery. 2016;78(3):448-457.

57. Merkus F, Verhoef J, Schipper N, Marttin E. Nasal mucociliary clearance as a factor in nasal drug delivery. Adv Drug Deliv Rev. 1998;29(1-2): $13-38$.

58. Fuller M, Meikle PJ, Hopwood JJ. Epidemiology of lysosomal storage diseases: an overview. In: Mehta A, Beck M, Sunder-Plassmann G, editors. Fabry Disease: Perspectives from 5 Years of FOS. Oxford, UK: Oxford PharmaGenesis; 2006.

59. Parenti G, Andria G, Ballabio A. Lysosomal storage diseases: from pathophysiology to therapy. Annu Rev Med. 2015;66:471-486.

60. Mehta A, Winchester B. Lysosomal Storage Disorders: A Practical Guide. Chichester, UK: Wiley; 2012.

61. Carvey PM, Hendey B, Monahan AJ. The blood-brain barrier in neurodegenerative disease: a rhetorical perspective. J Neurochem. 2009; 111(2):291-314.

62. Palmer AM. The role of the blood brain barrier in neurodegenerative disorders and their treatment. J Alzheimers Dis. 2011;24(4):643-656.

63. Cox TM. Innovative treatments for lysosomal diseases. Best Pract Res Clin Endocrinol Metab. 2015;29(2):275-311.

64. Barton NW, Brady RO, Dambrosia JM, et al. Replacement therapy for inherited enzyme deficiency macrophage-targeted glucocerebrosidase for Gaucher's disease. N Engl J Med. 1991;324(21):1464-1470.

65. Barton NW, Furbish FS, Murray GJ, Garfield M, Brady RO. Therapeutic response to intravenous infusions of glu- cocerebrosidase in a patient with Gaucher disease. Proc Natl Acad Sci U S A. 1990;87(5): 1913-1916.

66. Eng CM, Guffon N, Wilcox WR, et al. Safety and efficacy of recombinant human $\alpha$-galactosidase A replacement therapy in Fabry's disease. N Engl J Med. 2001;345(1):9-16.

67. Schiffmann R, Kopp JB, Austin HA 3rd, et al. Enzyme replacement therapy in Fabry disease: a randomized controlled trial. JAMA. 2001;285:2743-2749.

68. Van den Hout H, Reuser AJ, Vulto AG, Loonen MC, Cromme-Dijkhuis A, Van der Ploeg AT. Recombinant human $\alpha$-glucosidase from rabbit milk in Pompe patients. Lancet. 2000;356(9227):397-398.

69. Kakkis ED, Muenzer J, Tiller GE, et al. Enzyme-replacement therapy in mucopolysaccharidosis I. $N$ Engl J Med. 2001;344(3): $182-188$.

70. Muenzer J, Wraith JE, Beck M, et al. A phase II/III clinical study of enzyme replacement therapy with idursulfase in mucopolysaccharidosis II (Hunter syndrome). Genet Med. 2006;8(8):465-473.

71. Harmatz P, Giugliani R, Schwartz I, et al. Enzyme replacement therapy for mucopolysaccharidosis VI: a phase 3, randomized, double-blind, placebo-controlled, multinational study of recombinant human $\mathrm{N}$-acetylgalactosamine 4-sulfatase (recombinant human arylsulfatase B or rhASB) and follow-on, open-label extension study. J Pediatr. 2006;148:533-539.

72. Grubb JH, Vogler C, Levy B, Galvin N, Tan Y, Sly WS. Chemically modified $\beta$-glucuronidase crosses blood-brain barrier and clears neuronal storage in murine mucopolysaccharidosis VII. Proc Natl Acad Sci U S A. 2008;105:2616-2621.

73. Huynh HT, Grubb JH, Vogler C, Sly WS. Biochemical evidence for superior correction of neuronal storage by chemically modified enzyme in murine mucopolysaccharidosis VII. Proc Natl Acad Sci U S A. 2012;109:17022-17027.

74. Boado RJ, Zhang Y, Zhang Y, Xia CF, Wang Y, Pardridge WM. Genetic engineering, expression, and activity of achimeric monoclonal antibodyavidin fusion protein for receptor-mediated delivery of biotinylated drugs in humans. Bioconjug Chem. 2012;19:731-739.

75. Osborn MJ, McElmurry RT, Peacock B, Tolar J, Blazar BR. Targeting of the CNS in MPS-IH using a nonviral transferrin- $\alpha$-L-iduronidase fusion gene product. Mol Ther. 2008;16(8):1459-1466. 
76. Zhou QH, Boado RJ, Lu JZ, Hui EK, Pardridge WM. Brain-penetrating IgG-iduronate 2-sulfatase fusion protein for the mouse. Drug Metab Dispos. 2012;40(2):329-335.

77. Bockenhoff A, Cramer S, Wolte P, et al. Comparison of five peptide vectors for improved brain delivery of the lysosomal enzyme arylsulfatase A. J Neurosci. 2014;34(9):3122-3129.

78. Meng Y, Sohar I, Sleat DE, et al. Effective intravenous therapy for neurodegenerative disease with a therapeutic enzyme and a peptide that mediates delivery to the brain. Mol Ther. 2014;22(3):547-553.

79. Kakkis E, McEntee M, Vogler C, et al. Intrathecal enzyme replacement therapy reduces lysosomal storage in the brain and meninges of the canine model of MPS I. Mol Genet Metab. 2004;83(1-2):163-174.

80. Dickson P, McEntee M, Vogler C, et al. Intrathecal enzyme replacement therapy: successful treatment of brain disease via the cerebrospinal fluid. Mol Genet Metab. 2007;91(1):61-68.

81. Auclair D, Finnie J, White J, et al. Repeated intrathecal injections of recombinant human 4-sulfatase remove dural storage in mature mucopolysaccharidosis VI cats primed with a short-course tolerisation regimen. Mol Genet Metab. 2010;99:132-141.

82. Auclair D, Finnie J, Walkley SU, et al. Intrathecal recombinant human 4-sulfatase reduces accumulation of glycosaminoglycans in dura of mucopolysaccharidosis VI cats. Pediatr Res. 2012;71:39-45.

83. Bobo RH, Laske DW, Akbasak A, Morrison PF, Dedrick RL, Oldfield EH. Convection-enhanced delivery of macromolecules in the brain. Proc Natl Acad Sci U S A. 1994;91(6):2076-2080.

84. Kroll RA, Pagel MA, Muldoon LL, Roman-Goldstein S, Neuwelt EA. Increasing volume of distribution to the brain with interstitial infusion: dose, rather than convection, might be the most important factor. Neurosurgery. 1996;38(4):746-752.

85. Siegal T, Rubinstein R, Bokstein F, et al. In vivo assessment of the window of barrier opening after osmotic blood-brain barrier disruption in humans. J Neurosurg. 2000;92(4):599-605.

86. Costantino L, Boraschi D. Is there a clinical future for polymeric nanoparticles as brain-targeting drug delivery agents? Drug Discov Today. 2012;17(7):367-378

87. Spencer BJ, Verma IM. Targeted delivery of proteins across the bloodbrain barrier. Proc Natl Acad Sci US A. 2007;104(18):7594-7599.

88. Patel T, Zhou J, Piepmeier JM, Saltzman WM. Polymeric nanoparticles for drug delivery to the central nervous system. Adv Drug Deliv Rev. 2012;64(7):701-705.

89. Onodera H, Takada G, Tada K, Desnick RJ. Microautoradiographic study on the tissue localization of liposome-entrapped or unentrapped $3 \mathrm{H}-$ labeled beta-galactosidase injected into rats. Tohoku J Exp Med. 1983;140(1):1-13.
90. Chu Z, Sun Y, Kuan CY, Grabowski GA, Qi X. Saposin C: neuronal effect and CNS delivery by liposomes. Ann N Y Acad Sci. 2005;1053: 237-246.

91. Thekkedath R, Koshkaryev A, Torchilin VP. Lysosome-targeted octadecyl-rhodamine B-liposomes enhance lysosomal accumulation of glucocerebrosidase in Gaucher's cells in vitro. Nanomedicine. 2013; 8(7):1055-1065.

92. Tosi G, Bortot B, Ruozi B, et al. Potential use of polymeric nanoparticles for drug delivery across the blood-brain barrier. Curr Med Chem. 2013;20(17):2212-2225.

93. Tosi G, Ruozi B, Belletti D, et al. Brain-targeted polymeric nanoparticles: in vivo evidence of different routes of administration in rodents. Nanomedicine. 2013;8(9):1373-1383.

94. Tosi G, Vilella A, Chhabra R, et al. Insight on the fate of CNS-targeted nanoparticles. Part II: intercellular neuronal cell-to-cell transport. J Control Release. 2014;177:96-107.

95. Vilella A, Tosi G, Grabrucker AM, et al. Insight on the fate of CNStargeted nanoparticles. Part I: Rab5-dependent cell- specific uptake and distribution. J Control Release. 2014;174:195-201.

96. Pardridge WM. Drug and gene targeting to the brain with molecular Trojan horses. Nat Rev Drug Discov. 2002;1(2):131-139.

97. Tomanin R, Zanetti A, Zaccariotto E, D’Avanzo F, Bellettato CM, Scarpa M. Gene therapy approaches for lysosomal storage disorders, a good model for the treatment of mendelian diseases. Acta Paediatr. 2012;101(7):692-701.

98. Prinz M, Priller J, Sisodia SS, Ransohoff RM. Heterogeneity of CNS myeloid cells and their roles in neurodegeneration. Nat Neurosci. 2011; 14(10):1227-1235.

99. Visigalli I, Delai S, Politi LS, et al. Gene therapy augments the efficacy of hematopoietic cell transplantation and fully corrects mucopolysaccharidosis type I phenotype in the mouse model. Blood. 2010;116(24):5130-5139.

100. Cassano M, Felippu A. Endoscopic treatment of cerebrospinal fluid leaks with the use of lower turbinate grafts: a retrospective review of 125 cases. Rhinology. 2009;47(4):362-368.

101. Kimple AJ, Leight WD, Wheless SA, Zanation AM. Reducing nasal morbidity after skull base reconstruction with the nasoseptal flap: free middle turbinate mucosal grafts. Laryngoscope. 2012; 122(9):1920-1924.

102. Gill SS, Patel NK, Hotton GR, et al. Direct brain infusion of glial cell line-derived neurotrophic factor in Parkinson disease. Nat Med. 2003; 9(5):589-595.

\section{Publish your work in this journal}

Drug Design, Development and Therapy is an international, peerreviewed open-access journal that spans the spectrum of drug design and development through to clinical applications. Clinical outcomes, patient safety, and programs for the development and effective, safe, and sustained use of medicines are the features of the journal, which

\section{Dovepress}

has also been accepted for indexing on PubMed Central. The manuscript management system is completely online and includes a very quick and fair peer-review system, which is all easy to use. Visit http://www.dovepress.com/testimonials.php to read real quotes from published authors. 